Volume: 64, Issue: 11, pp. 4919-4922, Nov. 2016

\section{Implementation of open boundary problems in photo-conductive antennas by using convolutional perfectly matched layers}

\author{
E. Moreno, Z. Hemmat, J.B. Roldán, M. F. Pantoja, A. R. \\ Bretones, S. G. García and R. Faez
}

\begin{abstract}
A method to simulate an open boundary problem within the Finite Differences Time Domain (FDTD) approach for the emission of photo-conductive antennas is presented here. For this purpose we use convolutional perfectly matched layers (CPML). In these devices, the semiconductor region, where transient currents are present in simulation time, is considered to be an "active" medium. This medium is extended virtually beyond its boundaries or the computational domain limits. We explain in this paper how to simulate the transient state of a semiconductor in a CPML region as well as the potential of the method developed to solve conventional practical applications.
\end{abstract}

Index Terms-Terahertz (THz), Emitters, Emitter PhotoConductive Antenna (E-PCA), Simulations of Open Boundary Problem (OBP), Convolutional Perfectly Matched Layers (CPML), Finite Differences Time Domain (FDTD) method.

\section{INTRODUCTION}

The conventional simulation techniques used in the analysis of other radiating devices are being revisited to be adapted for the new needs found in the terahertz regime [1], [2]. In this work, we explain how to simulate the transient state of a semiconductor using CPML, an essential step forward in the simulation of an entire emitter photo-conductive antenna (E-PCA). The simulation an entire E-PCA requires an electromagnetic model with multiscale features [3], [4]. Under certain conditions, it is possible to simulate an entire E-PCA with the FDTD method by means of a two-stage procedure. The first stage is carried out in a truncated domain of the device (see Fig. 1(a)) and the second one in the entire antenna (see Fig. 1(b)). The first stage output is used as the input of the second one.

This manuscript is focused on the development of a procedure to deal with the first stage of the simulation where Fig. 1(c) shows the truncated part of the device under study. In doing this we carefully deal with the calculation of the current densities inside the CPML. We model the current densities by using the drift-diffusion model which has a determined applicability frame (AppF) which imposes conditions and limitations. One of the limitations shows up when we neglect the spatial variation of external forces. This assumption leads to a constant electric field in the semiconductor. We take into consideration that the applied external electric field varies spatially; therefore, this would mean that the drift-diffusion model falls apart, mostly at the locations where the electric field changes abruptly. As consequence, the mesh grid size is considerably smaller than the one needed for sampling the maximum frequency value which we want to resolve. With this approach we can avoid the electric field discontinuities at some mesh nodes and in addition we have a problem with well marked multiscale features.

In [2] we confined the charge carriers by means of artificial

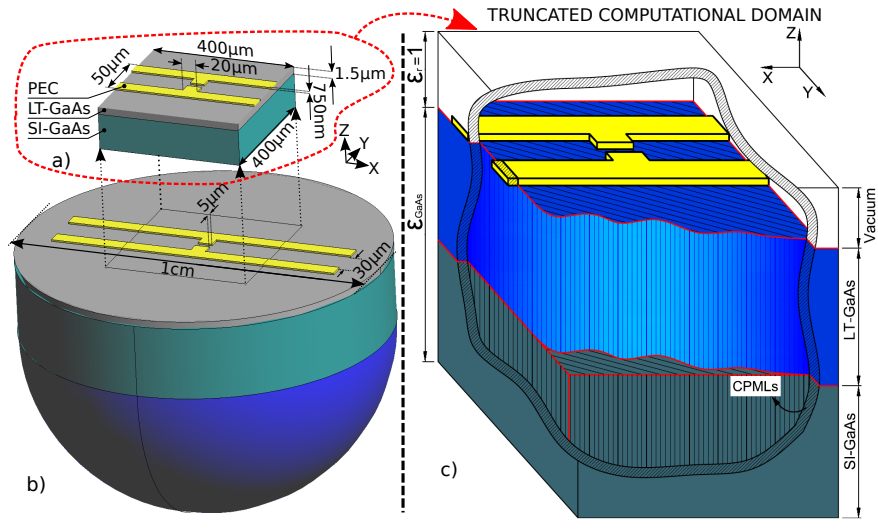

Fig. 1. a) A part of photo-conductive antenna consisting of the metal contacts and a region of the LT-GaAs layer. b) The whole photo-conductive antenna. c) The open boundary problem simulated in this paper. It corresponds with a chunk of the PCA shown in the figure 1(b).

boundary conditions. At first glance the truncation method in [2] and in this manuscript might look similar but it is quite different from the causality point of view. In [2] the emitter is a part of the real antenna that replaces the whole device and this emitter part is surrounded by vacuum. Therefore, the size of the part of the antenna simulated in [2] is close to the wavelength of the emitted radiation, and the diffraction effects are inevitable. That lack of causality and the diffraction effects are clearly present in [5] where we connect two computational domains so as to simulate the entire antenna. In this paper we extend the size of the emitter, which is much bigger than the wavelength by means of CPML, and also the degree of causality is much higher than in [2].

Accounting for all these issues, the paper is divided as follows: section II introduces the physical model, section III describes the computational domain, in section IV we validate the algorithms and analyze the results and finally in section $\mathrm{V}$ we draw some conclusions.

\section{THE PHYSICAL MODEL}

To model an E-PCA looking for a reasonable trade-off between simplicity and accuracy, we use the classical model expressed mathematically by a set of partial differential equations where Maxwell equations are coupled with the continuity equations. In this model, the current densities are modeled by drift-diffusion equations following previous results from [6], [2]. Prior to this new method we have already solved numerically this system of partial differential equations using FDTD [2]. Therefore, for the sake of clarity we summarize some essential points given in [2].

\section{A. The steady state}

We obtain the steady state with the same algorithms developed in [2] and insert the steady state solution into the CPML region. Thereby the semiconductor is placed inside of the CPML region (see Fig. 1(c)). Moreover, the mesh size is imposed by the dipole gap, because the maximum change of electric potential occurs in that area. We follow a rule in order to minimize the electric field discontinuity at the mesh nodes; 
this rule establishes that the change between two neighbor nodes in the gap must be less than $2 \%$ of the total applied electric potential.

\section{B. The transient regime}

The transient regime is described in two steps. In the first step, the laser hits the semiconductor and leads to the generation of pairs electron-hole. In the second step, the EPCA, due to recombination processes, radiates the rest of energy which has not yet been radiated along the generation (first step).

The coupled system of partial differential equations that describes the transient regime are the equations 3-6 introduced in [7]. In [2] we solved this system of differential equations and we introduced how to update the electric field $\vec{E}(\vec{r}, t)$ and the charge concentrations $p(\vec{r}, t)$ and $n(\vec{r}, t)$. Here we modify some of these expressions just at the CPML region.

The updating of the electric field components is analogous with respect to their spatial directions and we present here, and in section III, only the electric field updating process for the component in the $\mathrm{X}$-axis direction, whose mathematical form is:

$$
\begin{gathered}
\left.E_{x}\right|_{i+\frac{1}{2}, j, k} ^{m+\frac{1}{2}}=\left.\left.C a_{x}\right|_{i+\frac{1}{2}, j, k} ^{m} E_{x}\right|_{i+\frac{1}{2}, j, k} ^{m-\frac{1}{2}} \\
+\left.C b_{x}\right|_{i+\frac{1}{2}, j, k} ^{m}\left[\frac{\left.H_{z}\right|_{i+\frac{1}{2}, j+\frac{1}{2}, k} ^{m}-\left.H_{z}\right|_{i+\frac{1}{2}, j-\frac{1}{2}, k} ^{m}}{\delta_{y_{j}}}\right. \\
-\frac{\left.\left.H_{y}\right|_{i+\frac{1}{2}, j, k+\frac{1}{2}} ^{m}-\left.H_{y}\right|_{i+\frac{1}{2}, j, k-\frac{1}{2}} ^{m}-\left.J_{s x}\right|_{i+\frac{1}{2}, j, k} ^{m}\right]}{\delta_{z_{k}}}
\end{gathered}
$$

where $\left.J_{s x}\right|_{i+\frac{1}{2}, j, k} ^{m}=\left.\sigma_{i+\frac{1}{2}, j, k}^{m} E_{0 x}\right|_{i+\frac{1}{2}, j, k}$

$+q V_{T}\left(\frac{\left.\mu_{n}\right|_{i+\frac{1}{2}, j, k}\left(n_{i+1, j, k}^{m}-n_{i, j, k}^{m}\right)-\left.\mu_{p}\right|_{i+\frac{1}{2}, j, k}\left(p_{i+1, j, k}^{m}-p_{i, j, k}^{m}\right)}{\Delta x_{i}}\right)$ is the current density in the $\mathrm{X}$-axis direction. $\left.J_{s x}\right|_{i+\frac{1}{2}, j, k} ^{m}$ corresponds to equation 26 in [2], and $\left.C a_{x}\right|_{i+\frac{1}{2}, j, k} ^{m}$ and $\left.C b_{x}\right|_{i+\frac{1}{2}, j, k} ^{m}$ are defined in the expressions 33-36 in [2].

The charge carrier concentration, controlled by the continuity equations, updating process is given by:

$$
\begin{aligned}
n_{i, j, k}^{m+\frac{1}{2}} & =n_{i, j, k}^{m-\frac{1}{2}}+G_{i, j, k}^{m}-R_{i, j, k}^{m}+\left.q^{-1} \vec{\nabla} \cdot \vec{J}_{n T}\right|_{i, j, k} ^{m} \\
p_{i, j, k}^{m+\frac{1}{2}} & =p_{i, j, k}^{m-\frac{1}{2}}+G_{i, j, k}^{m}-R_{i, j, k}^{m}-\left.q^{-1} \vec{\nabla} \cdot \vec{J}_{p T}\right|_{i, j, k} ^{m}
\end{aligned}
$$

We omit $n_{i, j, k}^{m+1}$ and $p_{i, j, k}^{m+1}$ because its updating has been already explained in [2]. In [2], equations 2-3 are written in a matrix form. This compact notation does not explain how the divergence of transient current is calculated as a diference $\vec{\nabla} \cdot \vec{J}_{n T}(\vec{r}, t)=\vec{\nabla} \cdot \vec{J}_{n}(\vec{r}, t)-\vec{\nabla} \cdot \vec{J}_{n 0}(\vec{r})$ and $\vec{\nabla} \cdot \vec{J}_{n T}(\vec{r}, t)=\vec{\nabla} \cdot \vec{J}_{n}(\vec{r}, t)-\vec{\nabla} \cdot \vec{J}_{n 0}(\vec{r})$.

Inside of the CPML region we remove the source current $\vec{J}_{s}=\overline{0}$, transforms the total transient current $\vec{J}_{T}(\vec{r}, t)$ into ${\overrightarrow{J^{\prime}}}_{T}(\vec{r}, t)=\left(\sigma(\vec{r}, t)+\sigma_{0}(\vec{r})\right) \vec{E}(\vec{r}, t)$. However we do so only in the Ampere-Maxwell equation, remaining the currents $\vec{J}_{n T}(\vec{r}, t)$ and $\vec{J}_{p T}(\vec{r}, t)$ the same in the continuity equations. Another issue is the generation rate, there is not generation in the CPML region $G(\vec{r}, t)=0$.

When we adapt the electric field component in the $\mathrm{X}$-axis expressed by equation 1 to the new differential equations set
3-6 in [7], taking into account that we work inside a CPML region, then, the updated electric field component of in the $\mathrm{X}$-axis is the following:

$$
\begin{aligned}
\left.E_{x}\right|_{i+\frac{1}{2}, j, k} ^{m+\frac{1}{2}} & =\left.\left.C a_{x}\right|_{i+\frac{1}{2}, j, k} ^{m} E_{x}\right|_{i+\frac{1}{2}, j, k} ^{m-\frac{1}{2}} \\
+\left.C b_{x}\right|_{i+\frac{1}{2}, j, k} ^{m} & {\left[\begin{array}{l}
\left.H_{z}\right|_{i+\frac{1}{2}, j+\frac{1}{2}, k} ^{m}-\left.H_{z}\right|_{i+\frac{1}{2}, j-\frac{1}{2}, k} ^{m} \\
\delta_{y_{j}} \kappa_{y_{j}}
\end{array}\right.} \\
& \left.-\frac{\left.H_{y}\right|_{i+\frac{1}{2}, j, k+\frac{1}{2}} ^{m}-\left.\left.H_{y}\right|_{i+\frac{1}{2}, j, k-\frac{1}{2}} ^{m}\right|_{i+\frac{1}{2}, j, k} ^{m}-\left.\Psi_{E_{x z}}\right|_{i+\frac{1}{2}, j, k} ^{m}}{\delta_{z_{k}} \kappa_{z_{k}}}\right]
\end{aligned}
$$

The coefficients $\left.C a_{x}\right|_{i+\frac{1}{2}, j, k} ^{m}$ and $\left.C b_{x}\right|_{i+\frac{1}{2}, j, k} ^{m}$ do not change, therefore, we calculate them with the same expressions (3336) defined in [2]. The terms $\kappa_{y_{j}}, \kappa_{z_{k}},\left.\Psi_{E_{x y}}\right|_{i+\frac{1}{2}, j, k} ^{m}$, and $\left.\Psi_{E_{x z}}\right|_{i+\frac{1}{2}, j, k} ^{m}$ are fully explained in the literature [8].

In the CPML region the updatimg of charge carrier concentration is described by equations 2-3 but the diference is that $G_{i, j, k}^{m}=0$. In addition, we have to apply Neumann boundary conditions to the charge carrier concentration, only in Z-axis, as follows:

$$
\left.\frac{\partial w}{\partial \hat{n}}\right|_{\text {int. }}=0
$$

where $w \equiv \mathrm{n}$ or $\mathrm{p}$ and the subscript int. means the interface between LT-GaAs and any other media (SI-GaAs, Vacuum or metal contacts) along the $\mathrm{Z}$ direction.

On the $\mathrm{XZ}$ and $\mathrm{YZ}$ geometric planes, just at the boundary of the LT-GaAs on the outest surface of the CPML, we do not update the transient charge carrier concentration. Hence, $n(\vec{r}, t)$ and $p(\vec{r}, t)$ in all these locations are null in the entire simulation.

\section{THE COMPUTATIONAL DOMAIN}

We validated the algorithms developed in the previous section with a simulation where a large and square computational domain (LCD) surrounded by artificial boundary conditions

\begin{tabular}{|c|c|c|c|c|}
\hline Coordinates & $\mathrm{X}(\mu \mathrm{m})$ & $\mathrm{Y}(\mu \mathrm{m})$ & Coordinates & $\mathrm{Z}(\mu \mathrm{m})$ \\
\hline \hline $1,6,11,16,21$ & 0 & 0 & $1,2,3,4,5$ & -2 \\
\hline $2,7,12,17,22$ & 0 & 25 & $6,7,8,9,10$ & -1.5 \\
\hline $3,8,13,18,23$ & 50 & 25 & $11,12,13,14,15$ & -0.75 \\
\hline $4,9,14,19,24$ & 50 & 0 & $16,17,18,19,20$ & 0 \\
\hline $5,10,15,20,25$ & 25 & 5 & $21,22,23,24,25$ & -0.5 \\
\hline
\end{tabular}

PROBES AND THEIR COORDINATES REFERENCED TO THE ORIGIN $P_{0}$.

was considered, the same described in [2]. Illustration 2(a) corresponds to the LCD, it has a side length of $400 \mu \mathrm{m}$. In the LCD we implement CPML only in the geometric XY plane. The illustrations 1(c) and 2(b) correspond to the truncated computational domain (TCD) taken from LCD. This square TCD has a side length of $150 \mu m$ and it is surrounded by CPML. In both domains $P_{0}$ is the origin and source of the radiation. The positions of the truncated boundaries with respect to the origin $P_{0}$ must be such that the generation rate reduces its value at least in $5 \mathrm{~dB}$ with respect to the maximum 


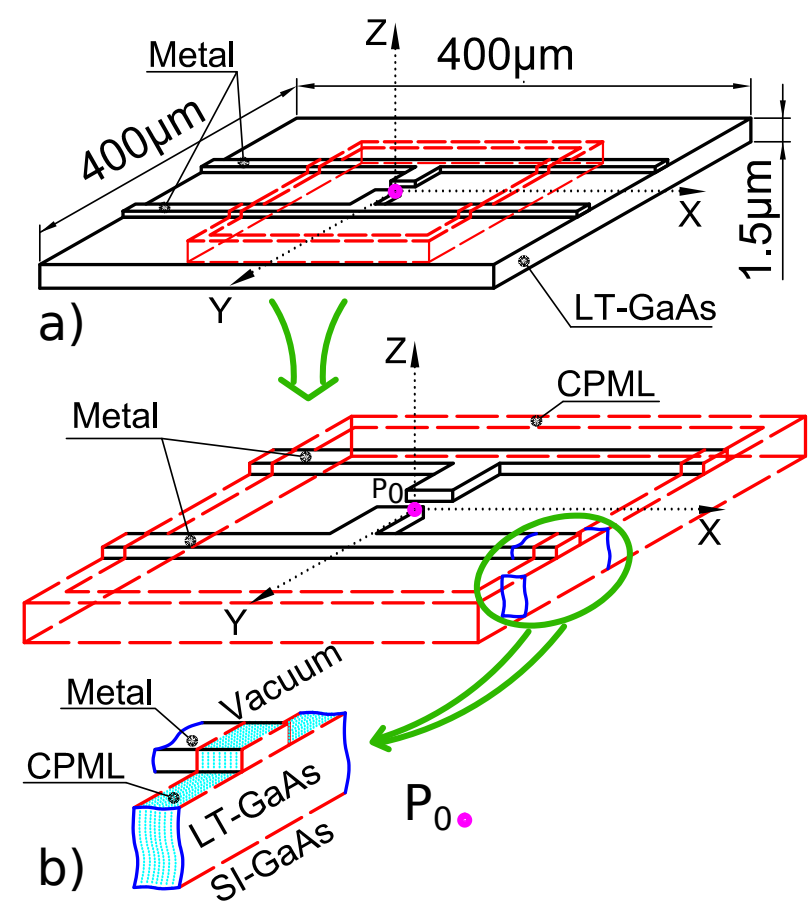

Fig. 2. Computational domains. The upper illustrations in a) and b) show the large computational domain while the bottom ones in a) and b) sketch the truncated computational domain.

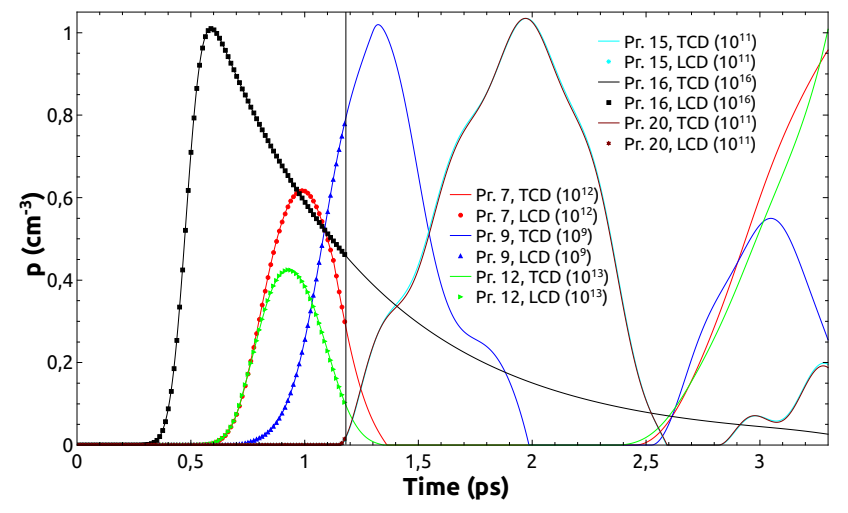

(a)

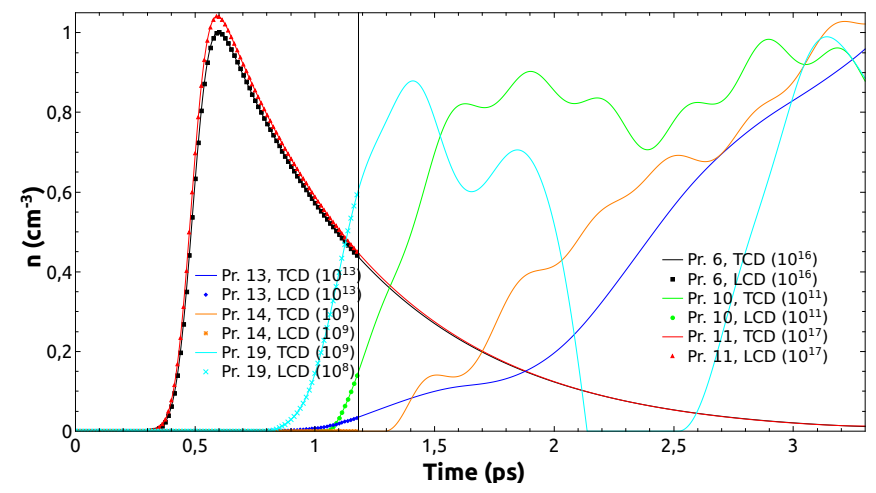

(b)

Fig. 3. a) Comparison between LCD and LCD for a) hole concentration and b) electron concentration. The relative error between LCD and TCD does not exceeded $5 \%$ in all cases.

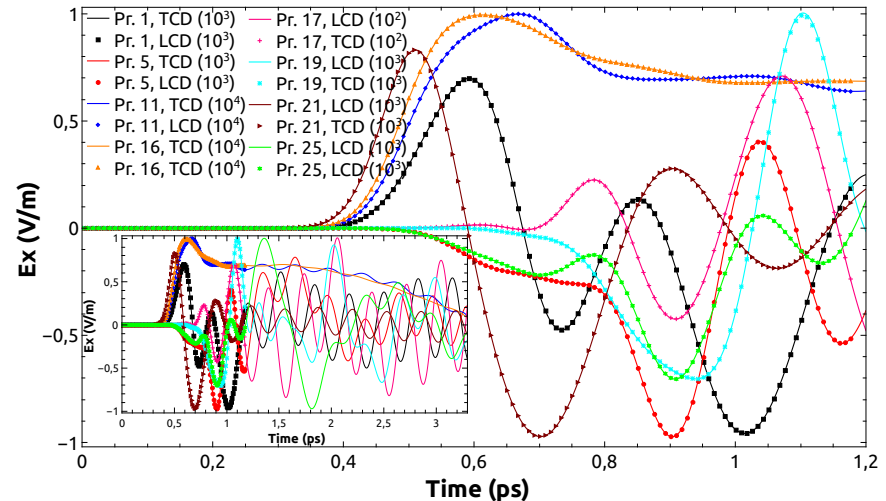

(a)

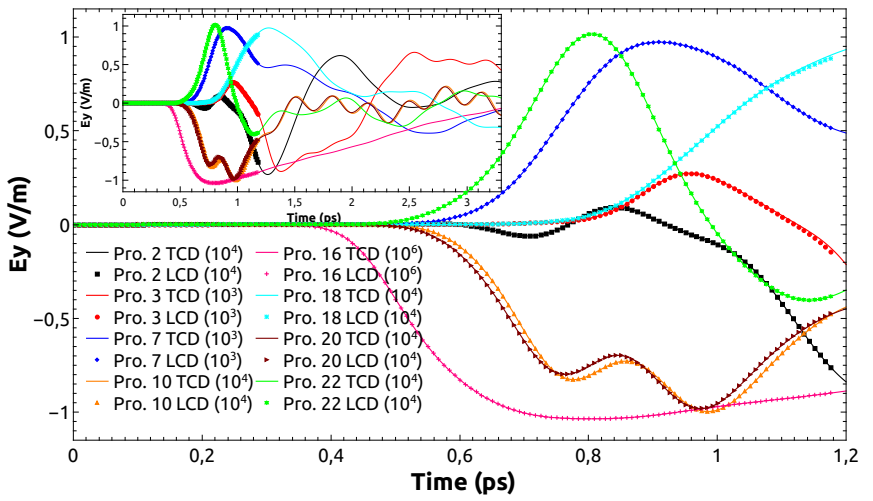

(b)

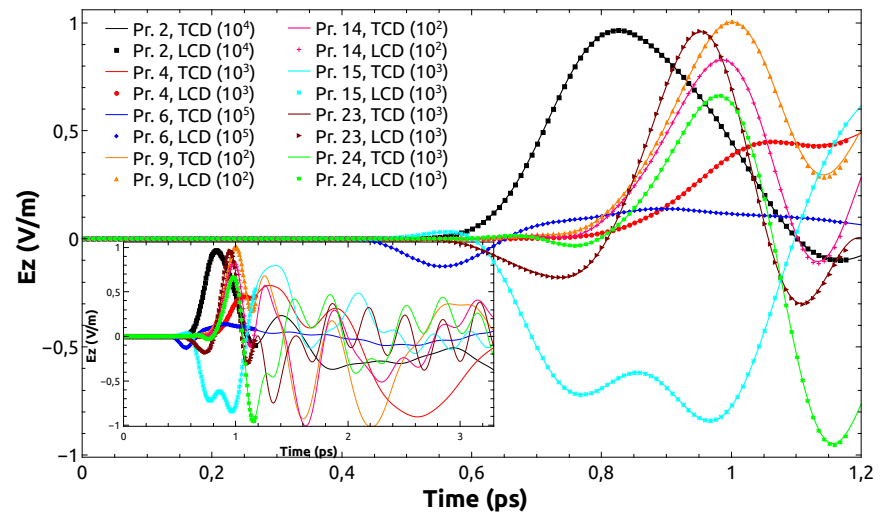

(c)

Fig. 4. a) Comparison between LCD and LCD for a) Electric field component in the X-axis, b) Electric field component in the Y-axis and c) Electric field component in the Z-axis. The relative error between LCD and TCD does not exceeded $5 \%$ in all cases.

value reached just at $P_{0}$.

The table I contains the coordinates of probe locations in relation to the origin $P_{0}$ and specifies the coordinates for each probe. The farthest away probe from the origin marks the simulation duration for the LCD. The distance between $P_{0}$ and that probe is $50 \mu \mathrm{m}$.

To avoid the measurement perturbations in the probes by the reflected field at the boundaries, we stop the simulation at LCD just before that event occurs. The simulation duration in the LCD is $t_{L C D}=1.167 \mathrm{ps}$. During the simulation $\frac{5 t_{L C D}}{7}$, in which we compare the measurements of the values in the 


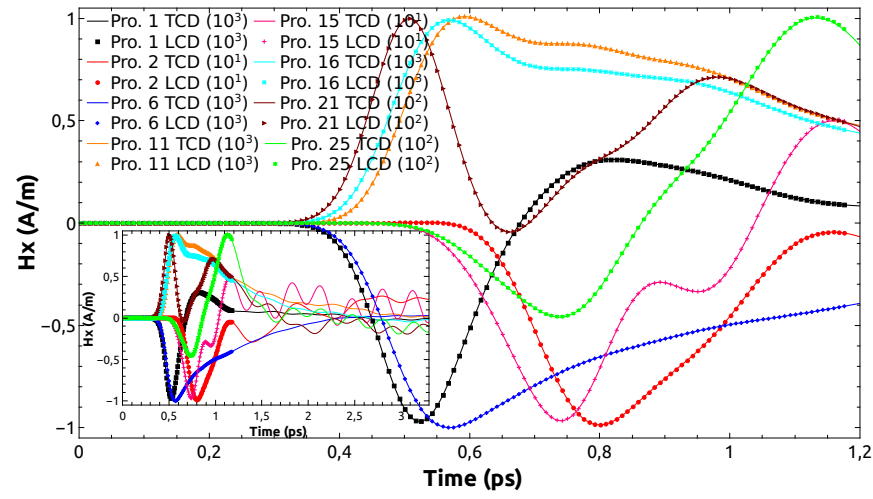

(a)

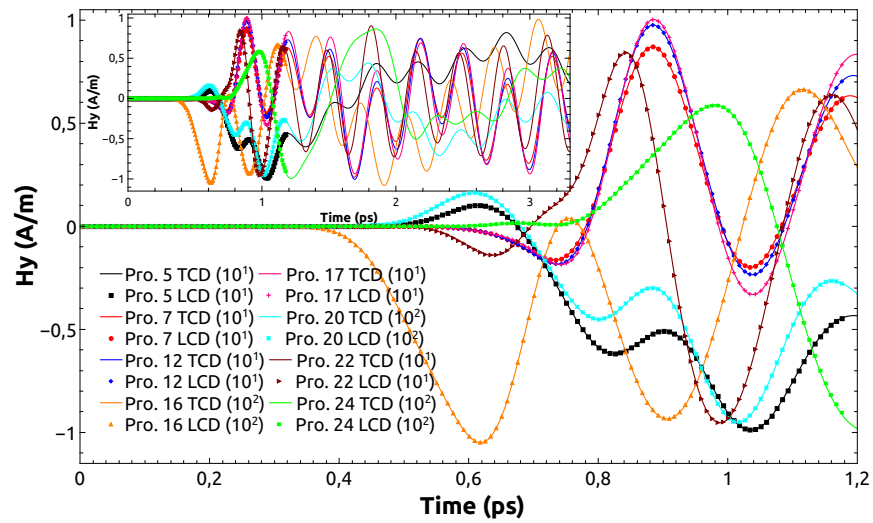

(b)

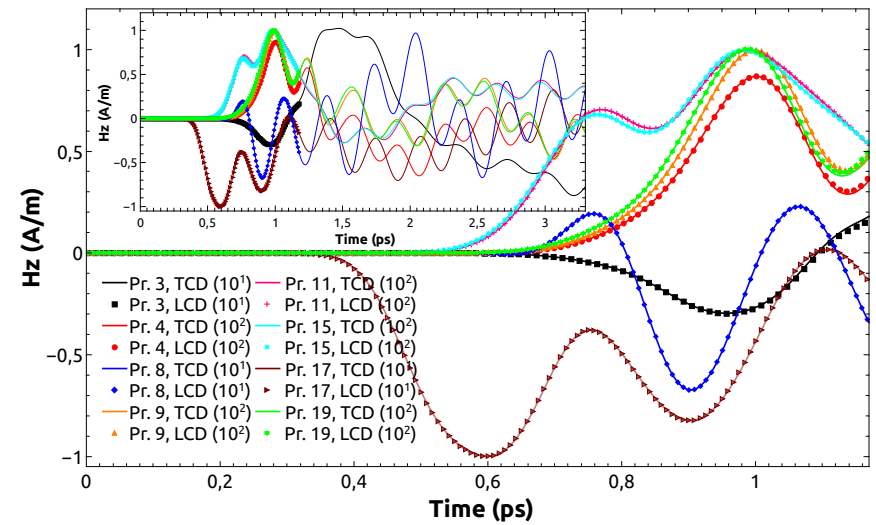

(c)

Fig. 5. a) Comparison between LCD and LCD for a) Magnetic field component in the $\mathrm{X}$-axis, b) Magnetic field component in the Y-axis and c) Magnetic field component in the Z-axis. The relative error between LCD and TCD does not exceeded 5\% in all cases.

probes between LCD and TCD, we compare clean profiles of LCD.

There is not a significant difference between the simulation speed of the LCD and the TCD achieving similar rate of Mcells per second.

\section{RESULTS}

We have compared the electric field, the magnetic field and the charge carrier concentration in several points of the computational domains, between LCD and TCD, which keep the same geometric locations from $P_{0}$ in both domains. After
$0.3 p s$ of simulation, and since that time to the simulation end in the LCD at time 1.167ps; the fields and charges recorded by the probes in the TCD are affected by the reflections in the CPML. That $0.867 p s$ interval is used for the validation of the algorithms. The simulation time for TCD is 3.3ps. We extended the simulation during this time to be sure that there are not numerical instabilities caused due to our new simulation approach. Figures 3(a) and 3(b) compare the electron and hole concentration at the probes 6-16,19 and 20. Figures 4(a), 4(b), 4(c) compare the $\mathrm{X}, \mathrm{Y}$ and $\mathrm{Z}$ electric field components. Thereby, figures 5(a), 5(b), 5(c) compare the $X$, $\mathrm{Y}$ and $\mathrm{Z}$ magnetic field components.

The agreement achieved and shown in the Figures described above is remarkably accurate.

\section{CONCLusions}

In this work a method to simulate a semiconductor into the CPML region is presented. We have validated the algorithms developed by comparing the electric field, the magnetic field and the charge carrier concentrations obtained by using two different simulations approaches. These two simulations schemes are carried out in a large computational domain, without CPML, and in a truncated computational domain, where there exists a clear CPML influence in the results. The results obtained demonstrate that the method is stable, works well and the output is reasonably accurate.

\section{REFERENCES}

[1] N. Burford and M. El-Shenawee, "Computational modeling of plasmonic thin-film terahertz photoconductive antennas," J. Opt. Soc. Am. B, vol. 33 , pp. 748-759, Apr 2016.

[2] E. Moreno, M. Pantoja, S. Garcia, A. Bretones, and R. Martin, "TimeDomain Numerical Modeling Of THz Photoconductive Antennas," Terahertz Science and Technology, IEEE Transactions on, vol. PP, no. 99, pp. 1-11, 2014.

[3] K. P. Biwojno, "Embedding of fine features in multi-scale electromagnetic models," Master's thesis, University of Nottingham, 2006.

[4] O. Ozgun and M. Kuzuoglu, "Numerical Solution of Multi-scale Electromagnetic Boundary Value Problems by Utilizing Transformation-Based Metamaterials," in Computational science ans its applications - ICCSA 2011, 2011.

[5] M. F. Pantoja, S. G. García, E. Moreno, A. R. Bretones, and R. G. Martín, "Full-wave Simulation of THz Photoconductive Antennas," in 39th International Conference on Infrared, Millimeter, and Terahertz Waves, 2014.

[6] J. Zhang, W.-R. Ng, M. Tuo, M. Liang, D. Golish, M. Gehm, and H. Xin, "Theoretical and experimental study of a terahertz time-domain spectrometer based on photoconductive antenna," in Radio Science Meeting (USNC-URSI NRSM), 2014 United States National Committee of URSI National, pp. 1-1, Jan 2014.

[7] E. Moreno, Z. Hemmat, J. B. Roldán, M. F. Pantoja, A. R. Bretones, and S. G. García, "Time-domain numerical modeling of terahertz receivers based on photoconductive antennas," J. Opt. Soc. Am. B, vol. 32, pp. 2034-2041, Oct 2015.

[8] A. Taflove and S. C. Hagness, Computational Electrodynamics The FiniteDifferences Time Domain Method. 2005. 